\title{
CORRECTIONS
}

\section{Author Correction: Using and producing publicly available genomic data to accelerate discovery in nephrology}

\section{Matthew G. Sampson (D) and Hyun Min Kang}

Nature Reviews Nephrology (2019) https://doi.org/10.1038/s41581-019-0166-z Published online 10 June 2019

The original article published online incorrectly listed the link to the CKDGEN consortium as https://fox.nhlbi.nih.gov/CKDGen/. This has been corrected in the HTML and PDF versions of the manuscript and replaced with the correct link: http://ckdgen.imbi.uni-freiburg.de.

https://doi.org/10.1038/s41581-019-0186-8 I Published online 30 July 2019 